J. R. Choike

Nagoya Math. J.

Vol. 49 (1973), 77-89

\title{
ON THE DISTRIBUTION OF VALUES OF FUNCTIONS IN THE UNIT DISK
}

\author{
JAMES R. CHOIKE*
}

\section{Introduction.}

Let $f(z)$ be a function analytic and bounded, $|f(z)|<1$, in $|z|<1$. Then, by Fatou's theorem the radial limit $f^{*}\left(e^{i \theta}\right)=\lim _{r \rightarrow 1} f\left(r e^{i \theta}\right)$ exists almost everywhere on $|z|=1$. Seidel [8, p. 208] and Calderón, GonzálezDomínguez, and Zygmund [1] (see also [9, pp. 281-282]) proved the following: if $f^{*}\left(e^{i \theta}\right)$ is of modulus 1 almost everywhere on an arc $a<\theta<b$ of $|z|=1$, then either $f(z)$ is analytically continuable across this arc or the values $f^{*}\left(e^{i \theta}\right), a<\theta<b$, cover the circumference $|w|=1$ infinitely many times. In this paper we shall be primarily concerned with the behavior of $f^{*}\left(e^{i \theta}\right)$ on each side of a singular point $P=e^{i \theta_{0}}, a<\theta_{0}<b$, for $f(z)$.

\section{One-side Limits.}

We shall say that $f(z)$ has a right-sided (left-sided) limit at $e^{i \theta_{0}}$ if there is a positive number $\delta$ such that $f^{*}\left(e^{i \theta}\right)$ exists and is continuous for all $\theta, \theta_{0}-\delta \leq \theta \leq \theta_{0}\left(\theta_{0} \leq \theta \leq \theta_{0}+\delta\right)$. We, now, state the first result of this paper which extends the theorem of Seidel and Calderón, GonzálezDomínguez, and Zygmund.

THEOREM 1. Let $f(z)$ be analytic and bounded, $|f(z)|<1$, in $|z|<1$. If $f^{*}\left(e^{i \theta}\right)$ is of modulus 1 almost everywhere on an arc $a<\theta<b$ of $|z|=1$ and if $P=e^{i \theta_{0}}, a<\theta_{0}<b$, is a singular point for $f(z)$, then either

i) the values of $f^{*}\left(e^{i \theta}\right), a<\theta<\theta_{0}$, cover $|w|=1$ infinitely many times and $f(z)$ has a left-sided limit at $e^{i \theta_{0}}$ of modulus 1 , or

ii) the values of $f^{*}\left(e^{i \theta}\right), \theta_{0}<\theta<b$, cover $|w|=1$ infinitely many times

Received April 10, 1972.

* This research was supported in part by a grant from the Oklahoma State University College of Arts and Sciences Office of Research and Graduate Studies. 
and $f(z)$ has a right-sided limit at $e^{i \theta_{0}}$ of modulus 1 , or

iii) the values of $f^{*}\left(e^{i \theta}\right)$ for both arcs $a<\theta<\theta_{0}$ and $\theta_{0}<\theta<b$, respectively, cover $|w|=1$ infinitely many times.

Proof. Without loss of generality, we may assume $a=2 \pi-\gamma, b=\gamma$, $\theta_{0}=0$ where $0<\gamma<\pi$.

Suppose $f^{*}\left(e^{i \theta}\right)$ assumes $\alpha,|\alpha|=1$, only finitely many times on the arc $2 \pi-\gamma<\theta<2 \pi$. Then, we may also assume, without loss of generality, that $f^{*}\left(e^{i \theta}\right)$ omits $\alpha$ on the arc $2 \pi-\gamma<\theta<2 \pi$.

Let $\zeta=L(w)$ be a bilinear transformation mapping $|w| \leq 1$ onto $\operatorname{Re}(\zeta) \geq 0$ such that $L(\alpha)=\infty$. The function $L(f(z))$ is analytic in $|z|<1$. The harmonic function $\operatorname{Re}(L(f(z)))$ is positive in $|z|<1$ with boundary values 0 almost everywhere on the arc $2 \pi-\gamma<\theta<2 \pi$. Thus,

$$
L(f(z))=\frac{1}{2 \pi} \int_{0}^{2 \pi} \frac{e^{i t}+z}{e^{i t}-z} d \mu(t)+i \operatorname{Im}(L(f(0)))
$$

where $\mu(t)$ is a bounded non-decreasing function $[0,2 \pi][9, \mathrm{p} .152]$. Let

$$
\begin{aligned}
u(r, \theta) & =\operatorname{Re}(L(f(z))) \\
& =\frac{1}{2 \pi} \int_{0}^{2 \pi} \frac{1-r^{2}}{1+r^{2}-2 r \cos (\theta-t)} d \mu(t)
\end{aligned}
$$

and

$$
\begin{aligned}
v(r, \theta) & =\operatorname{Im}(L(f(z))) \\
& =\frac{1}{\pi} \int_{0}^{2 \pi} \frac{r \sin (\theta-t)}{1+r^{2}-2 r \cos (\theta-t)} d \mu(t)+\operatorname{Im}(L(f(0))) .
\end{aligned}
$$

We wish, now, to examine the function $\mu(\theta)$. Since $\mu(\theta)$ is nondecreasing on $[0,2 \pi]$, its derivative $\mu^{\prime}(\theta)$ exists almost everywhere on $[0,2 \pi]$. The harmonic function $u(r, \theta)$ tends radially to $\mu^{\prime}(\theta)$ at every point of differentiability of $\mu(\theta)$. Since $u(r, \theta)$ has boundary values 0 almost everywhere for $2 \pi-\gamma<\theta<2 \pi, \mu^{\prime}(\theta)=0$ almost everywhere on $2 \pi-\gamma<\theta<2 \pi$.

Suppose $\mu(\theta)$ is not absolutely continuous on $2 \pi-\gamma<\theta<2 \pi$. Notice that $\mu(\theta)$ is of bounded variation on $[0,2 \pi]$. Then one of the following is true: i) $\mu(\theta)$ is continuous and not identically constant on $(2 \pi-\gamma, 2 \pi)$, ii) there exists $\theta^{*}, 2 \pi-\gamma<\theta^{*}<2 \pi$, such that $\mu(\theta)$ is discontinuous at $\theta^{*}$. If i) is the case, then from a theorem in Saks [7, p. 128] it follows that there exists $\theta_{1}, 2 \pi-\gamma<\theta_{1}<2 \pi$, such that $\mu^{\prime}(\theta)$ exists and is infinite 
at $\theta=\theta_{1}$. Thus, $\lim _{r \rightarrow 1} u\left(r, \theta_{1}\right)=\mu^{\prime}\left(\theta_{1}\right)=+\infty$. This implies that $f^{*}\left(e^{i \theta_{1}}\right)$ $=\alpha$, which is a contradiction. If ii) is the case, then by a lemma of Lohwater [4, p. 244] $\lim _{r \rightarrow 1} u\left(r, \theta^{*}\right)=+\infty$. Again, we have a contradiction, namely, $f^{*}\left(e^{i \theta^{*}}\right)=\alpha$. Thus, it follows that $\mu(\theta)$ is absolutely continuous on $2 \pi-\gamma<\theta<2 \pi$.

Since $\mu^{\prime}(\theta)=0$ almost everywhere on $2 \pi-\gamma<\theta<2 \pi, \mu(\theta)$ is constant on $2 \pi-\gamma<\theta<2 \pi$. Therefore, $L(f(z))$ is analytic at each point $e^{i \theta}, 2 \pi-\gamma<\theta<2 \pi$, and, in particular, we have

$$
L\left(f\left(e^{i \theta}\right)\right)=\frac{1}{2 \pi} \int_{0}^{2 \pi-r} \frac{e^{i t}+e^{i \theta}}{e^{i t}-e^{i \theta}} d \mu(t)+i \operatorname{Im}(L(f(0)))
$$

on $2 \pi-\gamma<\theta<2 \pi$. Hence, $f(z)$ is analytic at each point $e^{i \theta}, 2 \pi-\gamma<$ $\theta<2 \pi$, and $\left|f\left(e^{i \theta}\right)\right|=1$ at each $e^{i \theta}, 2 \pi-\gamma<\theta<2 \pi$.

Let $\varepsilon$ be a sufficiently small positive number. Since

$$
\begin{aligned}
v(1, \theta) & =\operatorname{Im}(L(f(0)))+\frac{1}{2 \pi} \int_{0}^{2 \pi-r} \frac{\sin (\theta-t)}{1-\cos (\theta-t)} d \mu(t) \\
& =\operatorname{Im}(L(f(0)))+\frac{1}{2 \pi} \int_{0}^{2 \pi-r} \cot \frac{1}{2}(\theta-t) d \mu(t) \\
& >\operatorname{Im}(L(f(0)))+\frac{1}{2 \pi} \int_{0}^{2 \pi-r} \cot \frac{1}{2}(\theta+\varepsilon-t) d \mu(t) \\
& =v(1, \theta+\varepsilon),
\end{aligned}
$$

it follows that as $\theta$ approaches $2 \pi$ through increasing values in $(2 \pi-\gamma, 2 \pi)$, $f\left(e^{i \theta}\right)$ moves along $|w|=1$ in a counterclockwise direction. Since $f\left(e^{i \theta}\right)$ omits $\alpha,|\alpha|=1$, on $(2 \pi-\gamma, 2 \pi), f\left(e^{i \theta}\right)$ cannot wind indefinitely around $|w|=1$ as $\theta$ approaches $2 \pi, 2 \pi-\gamma<\theta<2 \pi$. Hence, $f(z)$ has a rightsided limit $w_{1}$ of modulus 1 at $\theta_{0}=0$.

Suppose, next, that there exists a complex value $\beta,|\beta|=1$, such that $f^{*}\left(e^{i \theta}\right)$ assumes $\beta$ only finitely many times on the arc $0<\theta<\gamma$. Then, by the above argument, it follows that $f(z)$ has a left-sided limit $w_{2}$ of modulus 1 at $\theta_{0}=0$. By a well-known theorem of Lindelöf, $w_{1}=w_{2}$ [2, p. 43]. Another well-known theorem of Lindelöf [6, p. 75], then, implies that the cluster set of $f(z)$ at $P=1$ is $C(f, 1)=\left\{w_{1}\right\}$. But, since $P=1$ is a singular point for $f(z)$, a theorem of Seidel [2, p. 95] states $C(f, 1)$ $=\{|w| \leq 1\}$. We have a contradiction. Thus, $f(z)$ cannot have rightsided and left-sided limit at $P=1$ simultaneously. This completes our proof. 
A natural question which theorem 1 raises is this question: can functions $f(z)$ analytic and bounded, $|f(z)|<1$, in $|z|<1$ be found which exhibit each type of behavior as described in theorem 1 ? With regard to this question, we shall show by means of Blaschke products that theorem 1 is sharp in this sense. In fact, we shall give a necessary and sufficient condition for a Blaschke product to have a right-sided limit at $e^{i \theta_{0}}$.

\section{Blaschke Products.}

Let $\left\{a_{k}\right\}$ be a sequence of points in $|z|<1$ such that

$$
\sum_{k=1}^{\infty}\left(1-\left|a_{k}\right|\right)<+\infty \text {. }
$$

Then, the infinite product

$$
B(z)=\prod_{k=1}^{\infty} \frac{\left|a_{k}\right|}{a_{k}} \frac{a_{k}-z}{1-\bar{a}_{k} z}
$$

is a bounded, non-constant, holomorphic function in $|z|<1$. The function $B(z)$ is called a Blaschke product with zeros $\left\{a_{k}\right\}$. By Fatou's theorem the radial limit $B^{*}\left(e^{i \theta}\right)$ exists almost everywhere on $|z|=1$. It is also known that the modulus of $B^{*}\left(e^{i \theta}\right)$ is 1 almost everywhere on $|z|=1$. The following result of Frostman [3] (see also [2, p. 33-35]) gives a necessary and sufficient condition for $B^{*}\left(e^{i \theta_{0}}\right)$ to be of modulus 1 .

THEOREM A. Let $B(z)$ be a Blaschke product with zeros $\left\{a_{k}\right\}$. Then, a necessary and sufficient condition that $B(z)$ and all its partial products have radial limit of modulus 1 at $e^{i \theta_{0}}$ is the convergence of

$$
\sum_{k=1}^{\infty} \frac{1-\left|a_{k}\right|}{\left|e^{i \theta_{0}}-a_{k}\right|} .
$$

Remark. Geometrically, Frostman's condition implies that at most a finite number of zeros $\left\{a_{k}\right\}$ of $B(z)$ lie in any Stolz angle at $e^{i \theta_{0}}$.

For further properties of Blaschke products we refer the reader to [2, p. 28-38] or [9, p. 271-285].

THEOREM 2. Let $B(z)$ be a Blaschke product with zeros $\left\{a_{k}\right\}$ which have $e^{i \theta_{0}}$ as a limit point and which lie in a Stolz angle at $e^{i \theta_{0}}$. Then, for each $\delta, 0<\delta<\pi / 2$, the values of $B^{*}\left(e^{i \theta}\right)$ for the arcs $\theta_{0}-\delta<\theta<\theta_{0}$ and $\theta_{0}<\theta<\theta_{0}+\delta$, respectively, cover $|w|=1$ infinitely many times. 
Proof. Suppose $B(z)$ had either a right-sided or a left-sided limit at $e^{i \theta_{0}}$. This limit would, of course, be of modulus 1 . Then, by a theorem of Lindelöf, $B(z)$ would have angular limit at $e^{i \theta_{0}}$ of modulus 1 . But, this cannot happen, since the sequence $\left\{a_{k}\right\}$, by assumption, lies in a Stolz angle at $e^{i \theta_{0}}$. Thus, theorem 2 follows from theorem 1.

We, now, state the main result of this section.

THEOREM 3. Let $B(z)$ be a Blaschke product with zeros $\left\{a_{k}\right\}$. Then, $B(z)$ and all its partial products have a right-sided limit of modulus 1 at $e^{i \theta_{0}}$ if and only if

$$
\sum_{k=1}^{\infty} \frac{1-\left|a_{k}\right|}{\left|e^{i \theta_{0}}-a_{k}\right|}<+\infty
$$

and there exist positive numbers $\delta$ and $\varepsilon, \varepsilon<1$, such that there are no zeros $\left\{a_{k}\right\}$ in the region

$$
\Delta=\left\{z|1-\varepsilon<| z \mid<1, \theta_{0}-\delta<\arg (z)<\theta_{0}\right\} .
$$

Proof. Utilizing the proper rotation of $|z|<1$, we may assume that $\theta_{0}=0$. Suppose the zeros $\left\{a_{k}\right\}$ of $B(z)$ satisfy:

$$
\sum_{k=1}^{\infty} \frac{1-\left|a_{k}\right|}{\left|1-a_{k}\right|}<+\infty
$$

and there exist positive numbers $\delta$ and $\varepsilon, \varepsilon<1$, such that

$$
\left\{a_{1}, a_{2}, a_{3}, \cdots\right\} \cap\{z|1-\varepsilon<| z \mid<1,-\delta<\arg (z)<0\}=\emptyset .
$$

Choose $\delta$ so that $0<\delta<\pi / 2$. Let $\left\{a_{m_{j}}\right\}$ be the set of zeros $\left\{a_{k}\right\}$ of $B(z)$ lying in $\{z|| z \mid<1,0 \leq \arg (z) \leq \pi / 2\}$. Let $\left\{a_{n_{j}}\right\}$ be the remaining set of zeros $\left\{a_{k}\right\}$ of $B(z)$. From theorem $A$, (1) and (2), it follows that the radial limit $B^{*}\left(e^{i \theta}\right)$ exists and is of modulus 1 for all $\theta,-\delta / 2 \leq \theta \leq 0$. In order to prove that conditions (1) and (2) are sufficient for $B(z)$ to have a right-sided limit at $z=1$ it suffices to show that $\arg \left(B^{*}\left(e^{i \theta}\right)\right)$ is continuous for $\theta,-\delta / 2 \leq \theta \leq 0$. To do this we shall prove that for $k$ sufficiently large

$$
\left|\arg \left(\frac{\left|a_{k}\right|}{a_{k}} \frac{a_{k}-r e^{i \theta}}{1-\bar{a}_{k} r e^{i \theta}}\right)\right|
$$

is dominated by positive numbers $M_{k}$ whose sum forms a convergent series. By virtue of this, we assume that $\left\{a_{m_{j}}\right\}$ and $\left\{a_{n_{j}}\right\}$ are both sub- 
sequences of $\left\{a_{k}\right\}$.

It is clear that

$$
\begin{aligned}
\left|\arg \left(\frac{\left|a_{k}\right|}{a_{k}} \frac{a_{k}-r e^{i \theta}}{1-\bar{a}_{k} r e^{i \theta}}\right)\right| & =\left|\arg \left(\frac{\bar{a}_{k}\left(a_{k}-r e^{i \theta}\right)}{1-\bar{a}_{k} r e^{i \theta}}\right)\right| \\
& =\left|\arg \left(1-\frac{1-\left|a_{k}\right|^{2}}{1-\bar{a}_{k} r e^{i \theta}}\right)\right| \\
& =\left|\arcsin \frac{\left(1-\left|a_{k}\right|^{2}\right) r\left(\beta_{k} \cos \theta-\alpha_{k} \sin \theta\right)}{\left|\bar{a}_{k}\right|\left|a_{k}-r e^{i \theta}\right|\left|1-\bar{a}_{k} r e^{i \theta}\right|}\right| \\
& =\arcsin \frac{\left(1-\left|a_{k}\right|^{2}\right) r\left|\beta_{k} \cos \theta-\alpha_{k} \sin \theta\right|}{\left|\bar{a}_{k}\right|\left|a_{k}-r e^{i \theta}\right|\left|1-\bar{a}_{k} r e^{i \theta}\right|}
\end{aligned}
$$

where $a_{k}=\alpha_{k}+i \beta_{k}$. Since $\operatorname{arc} \sin x \leq \pi x / 2$ for $0 \leq x \leq 1$, it suffices to show that, for $k$ sufficiently large, the argument of the arc sin is dominated by positive numbers whose sum is a convergent series.

We, first, consider the zeros $\left\{a_{n_{j}}\right\}$. Let

$$
d_{1}=\inf \left|\frac{1}{\bar{a}_{n_{j}}}-r e^{i \theta}\right|, \quad j=1,2,3, \cdots, \quad 1-\frac{\varepsilon}{2}<r<1, \quad \frac{-\delta}{2} \leq \theta \leq 0,
$$

and

$$
d_{2}=\inf \left|a_{n_{j}}-r e^{i \theta}\right|, \quad j=1,2,3, \cdots, \quad 1-\frac{\varepsilon}{2}<r<1, \quad-\frac{\delta}{2} \leq \theta \leq 0 .
$$

By (2), we have $d_{1}>0$ and $d_{2}>0$. Let $d=\min \left(d_{1}, d_{2}\right)$. If $k=n_{f}$, then, from the way in which $d$ was chosen,

$$
\begin{aligned}
\frac{\left(1-\left|a_{k}\right|^{2}\right) r\left|\beta_{k} \cos \theta-\alpha_{k} \sin \theta\right|}{\left|\bar{a}_{k}\right|\left|a_{k}-r e^{i \theta}\right|\left|1-\bar{a}_{k} r e^{i \theta}\right|} & \leq \frac{4\left(1-\left|a_{k}\right|\right)}{\left|\bar{a}_{k}\right|^{2}\left|a_{k}-r e^{i \theta}\right|\left|\frac{1}{\bar{a}_{k}}-r e^{i \theta}\right|} \\
& \leq \frac{4}{\left|\bar{a}_{k}\right|^{2} d^{2}}\left(1-\left|a_{k}\right|\right)
\end{aligned}
$$

for $1-\varepsilon / 2<r<1$ and $-\delta / 2 \leq \theta \leq 0$.

Next, we consider the zeros $\left\{a_{m_{j}}\right\}$. Let $L_{1}$ and $L_{2}$ be chords of $|z|<1$ drawn from $z=1$ inclined from the radius to $z=1$ by an angle of $\delta / 2$ and $\delta / 4$, respectively. Let $\Delta_{1}$ be the triangle with sides $L_{1}$ and the radii to the endpoints of the chord $L_{1}$. Let $\Delta_{2}$ be the triangle formed in the same way as $\Delta_{1}$, but, instead of $L_{1}$, we use the chord $L_{2}$. For $\theta,-\delta / 2<\theta<0$, let $\Delta_{2}(\theta)$ be the triangle obtained by rotating $\Delta_{2}$ 
through an angle of $\theta$ about its vertex $z=0$ (see figure 1). Let $L_{2}(\theta)$ be the side of $\Delta_{2}(\theta)$ which is a chord of $|z|<1$. From the construction, it is clear that

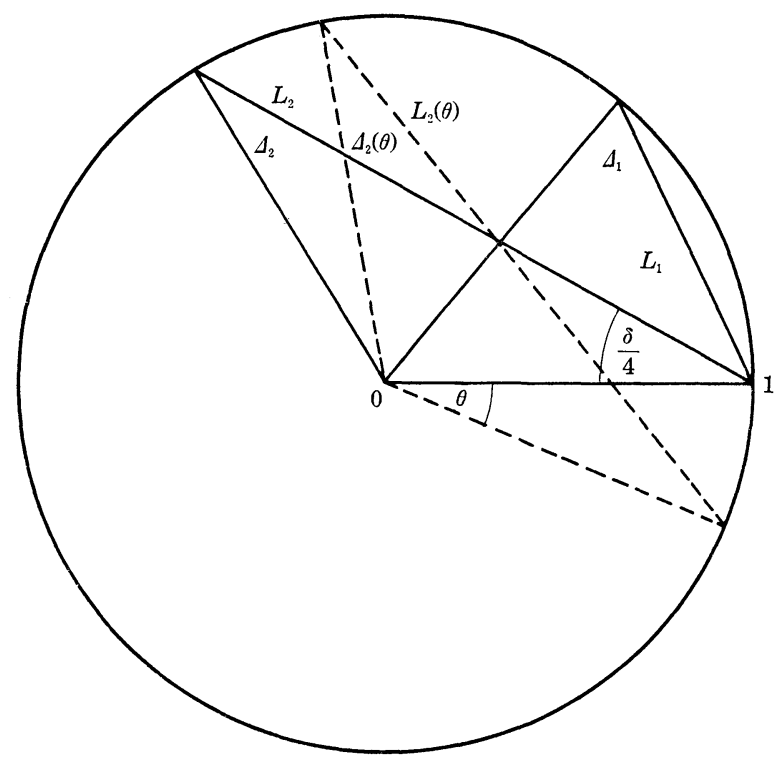

Fig. 1

$$
L_{2}(\theta) \cap L_{1}=\phi \text { for } \quad-\frac{\delta}{2}<\theta<0
$$

By (1) and the remark following theorem $\mathrm{A}$, there exists a positive integer $J$ such that $a_{m_{j}}$ lies outside $\Delta_{1}$ for $j>J$. Let $s(a, b)$ denote line segment joining the complex numbers $a$ and $b$. Then, we denote by $\phi_{j}(\theta)$ the angle formed at $e^{i \theta}$ by $s\left(0, e^{i \theta}\right)$ and $s\left(a_{m_{j}}, e^{i \theta}\right),-\delta / 2 \leq \theta \leq 0$. We lengthen the segment $s\left(a_{m_{j}}, e^{i \theta}\right)$ so that it is a chord $L_{j}^{\prime}(\theta)$ of $|z|<1$ (see figure 2). Then,

$$
L_{j}^{\prime}(\theta) \cap L_{1} \neq \emptyset
$$

for $j<J$ and $-\delta / 2 \leq \theta<0$. Thus, from (3) and (4) we have that the arc of $|z|=1$ cut off by $L_{2}(\theta)$ is greater than the arc of $|z|=1$ cut off by $L_{j}^{\prime}(\theta)$ for $j>J$ and $-\delta / 2 \leq \theta<0$. Thus,

$$
\pi-2 \phi_{j}(\theta)<\pi-\frac{\delta}{2}
$$




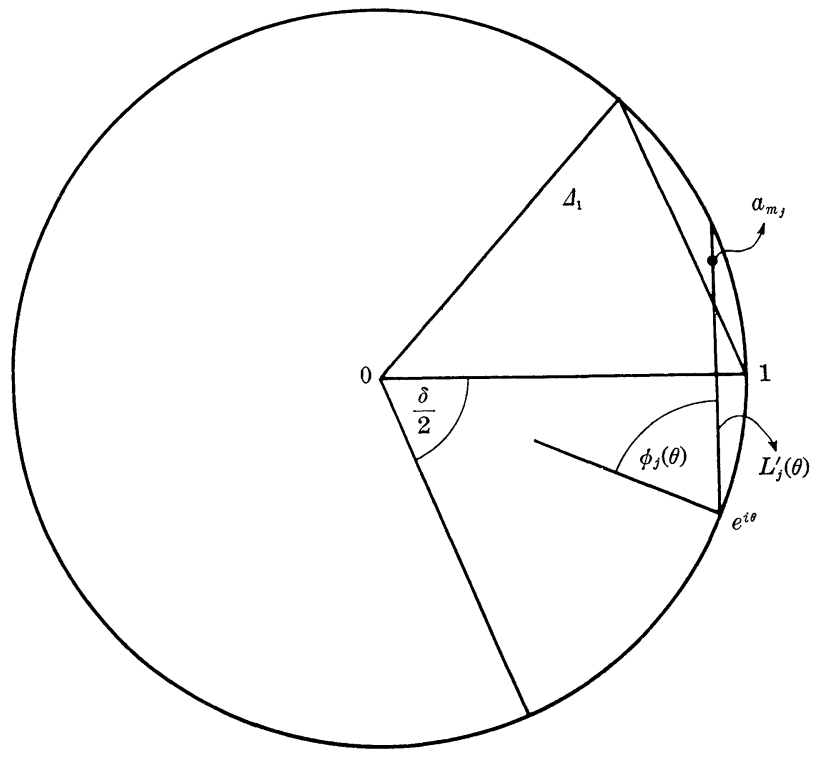

Fig. 2

for $j>J$ and $-\delta / 2 \leq \theta<0$. Also, since $a_{m_{j}}$ lies outside $\Delta_{1}$ for $j>J$, $\phi_{j}(0)>\delta / 2>\delta / 4$ for $j>J$. Thus, $\phi_{j}(\theta)>\delta / 4$ for $j>J$ and $-\delta / 2 \leq \theta \leq 0$. This implies

$$
\begin{aligned}
\left|a_{m_{j}}-r e^{i \theta}\right| & \geq\left|a_{m_{j}}-e^{i \theta}\right| \sin \phi_{j}(\theta) \\
& \geq\left|a_{m_{j}}-e^{i \theta}\right| \sin \frac{\delta}{4}
\end{aligned}
$$

for $j>J$ and $-\delta / 2 \leq \theta \leq 0$. But, for all $j$ and $-\delta / 2 \leq \theta \leq 0,-\sin \theta_{i} \leq$ $\left|a_{m_{j}}-e^{i \theta}\right|$. Thus,

$$
\begin{aligned}
\frac{-\sin \theta}{\left|a_{m_{j}}-r e^{i \theta}\right|} & \leq \frac{-\sin \theta}{\left|a_{m_{j}}-e^{i \theta}\right|} \frac{1}{\sin (\delta / 4)} \\
& \leq \frac{1}{\sin (\delta / 4)}
\end{aligned}
$$

for $j>J$ and $-\delta / 2 \leq \theta \leq 0$. Also,

$$
\operatorname{Im}\left(a_{m_{j}}\right)=\beta_{m_{j}} \leq\left|a_{m_{j}}-r e^{i \theta}\right|
$$

for all $j$ and $-\delta / 2 \leq \theta \leq 0$. Therefore,

$$
\frac{\beta_{m_{j}}-\sin \theta}{\left|a_{m_{j}}-r e^{i \theta}\right|} \leq 1+\frac{1}{\sin (\delta / 4)}=C<+\infty
$$


for $j>J$ and $-\delta / 2 \leq \theta \leq 0$.

Let $\gamma_{j}$ be the angle between $s\left(a_{m_{j}}, 1\right)$ and $s(0,1)$. Recalling that $a_{m_{j}}$ lies outside $\Delta_{1}$ for $j>J$, we have $\pi / 2>\gamma_{j}>\delta / 2$ for $j>J$ and $-\delta / 2 \leq \theta \leq 0$. Hence,

$$
\begin{aligned}
\left|\frac{1}{\bar{a}_{m_{j}}}-r e^{i \theta}\right| & \geq \operatorname{Im}\left(\frac{1}{\bar{a}_{m_{j}}}\right) \\
& \geq \operatorname{Im}\left(a_{m_{j}}\right) \\
& =\left|1-a_{m_{j}}\right| \sin \gamma_{j} \\
& \geq\left|1-a_{m_{j}}\right| \sin \frac{\delta}{2}
\end{aligned}
$$

for $j>J$ and $-\delta / 2 \leq \theta \leq 0$. Thus,

$$
\frac{1}{\left|1 / \bar{a}_{m_{j}}-r e^{i \theta}\right|} \leq \frac{1}{\left|1-a_{m_{j}}\right| \sin (\delta / 2)}
$$

for $j>J$ and $-\delta / 2 \leq \theta \leq 0$.

Using the estimates (5) and (6), we have, for $j>J,-\delta / 2 \leq \theta \leq 0$, and $0<r<1$,

$$
\begin{aligned}
\frac{\left(1-\left|a_{m_{j}}\right|^{2}\right) r\left|\beta_{m_{j}} \cos \theta-\alpha_{m_{j}} \sin \theta\right|}{\left|\bar{a}_{m_{j}}\right|^{2}\left|a_{m_{j}}-r e^{i \theta}\right|\left|1 / \bar{a}_{m_{j}}-r e^{i \theta}\right|} & \leq \frac{2\left(1-\left|a_{m_{j}}\right|\right)\left(\beta_{m_{j}}-\sin \theta\right)}{\left|\bar{a}_{m_{j}}\right|^{2}\left|a_{m_{j}}-r e^{i \theta}\right|\left|1 / \bar{a}_{m_{j}}-r e^{i \theta}\right|} \\
& \leq \frac{2 C}{\left|a_{m_{j}}\right|^{2} \sin (\delta / 2)} \frac{1-\left|a_{m_{j}}\right|}{\left|1-a_{m_{j}}\right|} .
\end{aligned}
$$

Thus, for a positive integer $K$ chosen sufficiently large, we have

$$
\left|\arg \left(\frac{\left|a_{k}\right|}{a_{k}} \frac{a_{k}-r e^{i \theta}}{1-\bar{a}_{k} r e^{i \theta}}\right)\right|= \begin{cases}C_{1}\left(1-\left|a_{k}\right|\right)=M_{k}, & \text { if } k=n_{j}, \\ C_{2} \frac{1-\left|a_{k}\right|}{\left|1-a_{k}\right|}=M_{k}, & \text { if } k=m_{j},\end{cases}
$$

for $k>K$ and $1-\varepsilon / 2<r<1,-\delta / 2 \leq \theta \leq 0$, where $C_{1}$ and $C_{2}$ are constants. Note that

$$
\sum_{k=K}^{\infty} M_{k} \leq C_{1} \sum_{k=1}^{\infty}\left(1-\left|a_{k}\right|\right)+C_{2} \sum_{k=1}^{\infty} \frac{1-\left|a_{k}\right|}{\left|1-a_{k}\right|}<+\infty .
$$

Note, also, that for any integer $K^{\prime}, K^{\prime}>K$,

$$
\begin{aligned}
\sum_{k=1}^{\infty}\left|\arg \left(\frac{\mid a_{k}}{a_{k}} \frac{\mid a_{k}-r e^{i \theta}}{1-\bar{a}_{k} r^{i \theta}}\right)\right| & \leq \sum_{k=1}^{K^{\prime}}\left|\arg \left(\frac{\left|a_{k}\right|}{a_{k}} \frac{a_{k}-r e^{i \theta}}{1-\bar{a}_{k} r e^{i \theta}}\right)\right|+\sum_{k=K^{\prime}+1}^{\infty} M_{k} \\
& \leq \sum_{k=1}^{K}\left|\arg \left(\frac{\left|a_{k}\right|}{a_{k}} \frac{a_{k}-r e^{i \theta}}{1-\bar{a}_{k} r e^{i \theta}}\right)\right|+\sum_{k=K+1}^{\infty} M_{k}
\end{aligned}
$$


for $1-\varepsilon / 2<r<1$ and $-\delta / 2 \leq \theta \leq 0$.

Choose an arbitrary, but fixed, point $r e^{i \theta}, 1-\varepsilon / 2<r<1$ and $-\delta / 2 \leq \theta \leq 0$. Let $\varepsilon_{0}$ be an arbitrary positive number. We can choose an integer $K^{\prime}>K$ sufficiently large that

$$
\left|\arg B\left(r e^{i \theta}\right)-\arg \prod_{k=1}^{K^{\prime}} \frac{\left|a_{k}\right|}{a_{k}} \frac{a_{k}-r e^{i \theta}}{1-\bar{a}_{k} r e^{i \theta}}\right|<\frac{\varepsilon_{0}}{2}
$$

and

$$
\sum_{k=K^{\prime}+1}^{\infty} M_{k}<\frac{\varepsilon_{0}}{2}
$$

Then,

$$
\begin{aligned}
\mid \arg B\left(r e^{i \theta}\right)- & \sum_{k=1}^{\infty} \arg \left(\frac{a_{k} \mid}{a_{k}} \frac{a_{k}-r e^{i \theta}}{1-\bar{a}_{k} r e^{i \theta}}\right) \mid \\
& \leq\left|\arg B\left(r e^{i \theta}\right)-\sum_{k=1}^{K^{\prime}} \arg \left(\frac{\left|a_{k}\right|}{a_{k}} \frac{a_{k}-r e^{i \theta}}{1-\bar{a}_{k} r e^{i \theta}}\right)\right|+\sum_{k=K^{\prime}+1}^{\infty} M_{k} \\
& \leq\left|\arg B\left(r e^{i \theta}\right)-\arg \prod_{k=1}^{K^{\prime}} \frac{\left|a_{k}\right|}{a_{k}} \frac{a_{k}-r e^{i \theta}}{1-\bar{a}_{k} r e^{i \theta}}\right|+\sum_{k=K^{\prime}+1}^{\infty} M_{k}<\varepsilon_{0}
\end{aligned}
$$

for $1-\varepsilon / 2<r<1$ and $-\delta / 2 \leq \theta \leq 0$. Since $\varepsilon_{0}$ is arbitrary, we have

$$
\arg B\left(r e^{i \theta}\right)=\sum_{k=1}^{\infty} \arg \left(\frac{\left|a_{k}\right|}{a_{k}} \frac{a_{k}-r e^{i \theta}}{1-\bar{a}_{k} r e^{i \theta}}\right)
$$

for $1-\varepsilon / 2<r<1$ and $-\delta / 2 \leq \theta \leq 0$. Also, this series converges uniformly, with $\theta$ fixed, in the region $1-\varepsilon / 2<r<1$ and $-\delta / 2 \leq \theta \leq 0$, and the uniform convergence implies

$$
\begin{aligned}
\arg B^{*}\left(e^{i \theta}\right) & =\lim _{r \rightarrow 1} \sum_{k=1}^{\infty} \arg \left(\frac{\left|a_{k}\right|}{a_{k}} \frac{a_{k}-r e^{i \theta}}{1-\bar{a}_{k} r e^{i \theta}}\right) \\
& =\sum_{k=1}^{\infty} \arg \left(\frac{\left|a_{k}\right|}{a_{k}} \frac{a_{k}-e^{i \theta}}{1-\bar{a}_{k} e^{i \theta}}\right)
\end{aligned}
$$

for $-\delta / 2 \leq \theta \leq 0$. Finally, we remark that

$$
\left|\arg \left(\frac{\left|a_{k}\right|}{a_{k}} \frac{a_{k}-e^{i \theta}}{1-\bar{a}_{k} e^{i \theta}}\right)\right| \leq M_{k}
$$

for $k>K$ and $-\delta / 2 \leq \theta \leq 0$. This implies that the series

$$
\sum_{k=1}^{\infty} \arg \left(\frac{\left|a_{k}\right|}{a_{k}} \frac{a_{k}-e^{i \theta}}{1-\bar{a}_{k} e^{i \theta}}\right)
$$


converges uniformly to $\arg B^{*}\left(e^{i \theta}\right)$ for $-\delta / 2 \leq \theta \leq 0$. Thus, $\arg B^{*}\left(e^{i \theta}\right)$ is continuous for $-\delta / 2 \leq \theta \leq 0$. This completes the proof of theorem 3 in one. direction.

It is clear that the same conclusion holds for all partial products of $B(z)$.

Conversely, let us assume that $B(z)$ has right-sided limit of modulus 1 at $e^{i \theta_{0}}$. Then, by a theorem of Lindelöf, $B(z)$ has angular limit of modulus 1 at $e^{i \theta_{0}}$, and, hence, radial limit of modulus 1 at $e^{i \theta_{0}}$. Thus, by theorem $\mathrm{A}$, the zeros $\left\{a_{k}\right\}$ of $B(z)$ satisfy the condition

$$
\sum_{k=1}^{\infty} \frac{1-\left|a_{k}\right|}{\left|e^{i \theta_{0}}-a_{k}\right|}<+\infty
$$

Since $B(z)$ has right-sided limit of modulus 1 at $e^{i \theta_{0}}$, there exists $\delta>0$ such that $B^{*}\left(e^{i \theta}\right)$ exists and is continuous for all $\theta, \theta_{0}-\delta \leq \theta \leq \theta_{0}$. Let $R=\left\{z|| z \mid<1, \theta_{0}-\delta<\arg (z)<\theta_{0}\right\}$. Now, in the sector $R$, we have that $B(z)$ is analytic and bounded. Moreover, $B(z)$ has radial limit of modulus 1 at $e^{i \theta_{0}}$ and right-sided limit of modulus 1 at $e^{i \theta_{0}}$. Thus, by another theorem of Lindelöf, $B(z)$ converges to a value of modulus 1 as $z$ tends to $e^{i \theta_{0}}, z \in R$. It follows that $R$ contains at most a finite number of zeros of $B(z)$. Thus, there exists $\varepsilon, 0<\varepsilon<1$, such that there are no zeros $\left\{a_{k}\right\}$ in the region

$$
\Delta=\left\{z|1-\varepsilon<| z \mid<1, \theta_{0}-\delta<\arg (z)<\theta_{0}\right\} .
$$

This completes the proof of theorem 3 .

A direct consequence of theorem 3 is the following theorem.

THEOREM 4. A necessary and sufficient condition for a Blaschke product $B(z)$ with zeros $\left\{a_{k}\right\}$ to have a right-sided limit of modulus 1 at $e^{i \theta_{0}}$ but not a left-sided limit at $e^{i \theta_{0}}$ is that the zeros $\left\{a_{k}\right\}$ satisfy the following properties:

i) $e^{i \theta_{0}}$ is a limit point of $\left\{a_{k}\right\}$,

ii) $\sum_{k=1}^{\infty} \frac{1-\left|a_{k}\right|}{\left|e^{i \theta_{0}}-a_{k}\right|}<+\infty$, and

iii) there exist positive numbers $\delta$ and $\varepsilon, \varepsilon<1$, such that there are no zeros $\left\{a_{k}\right\}$ in the region

$$
\Delta=\left\{z|1-\varepsilon<| z \mid<1, \theta_{0}-\delta<\arg (z)<\theta_{0}\right\} .
$$

Proof. Theorem 1 and theorem 3 imply that properties i), ii), and 
iii) are sufficient for $B(z)$ to have right-sided limit at $e^{i \theta_{0}}$ but not leftsided limit at $e^{i \theta_{0}}$. This follows easily once we notice that property i) implies that $e^{i \theta_{0}}$ is a singular point for $B(z)$ and properties ii) and iii) imply that $B(z)$ has right-sided limit at $e^{i \theta_{0}}$. The Blaschke product $B(z)$ cannot have left-sided limit at $e^{i \theta_{0}}$, otherwise we contradict theorem 1 .

To prove that properties i), ii), and iii) are necessary, let us suppose that $B(z)$ has right-sided limit of modulus 1 at $e^{i \theta_{0}}$, but not left-sided limit at $e^{i \theta_{0}}$. Thus, by theorem 3 , we have that

$$
\sum_{k=1}^{\infty} \frac{1-\left|a_{k}\right|}{\left|e^{i \theta_{0}}-a_{k}\right|}<+\infty
$$

and that there exist positive numbers $\delta$ and $\varepsilon, \varepsilon<1$, such that there are no zeros $\left\{a_{k}\right\}$ in the region

$$
\Delta=\left\{z|1-\varepsilon<| z \mid<1, \theta_{0}-\delta<\arg (z)<\theta_{0}\right\} .
$$

Since $B(z)$ does not have left-sided limit at $e^{i \theta_{0}}, P=e^{i \theta_{0}}$ is a singular point for $B(z)$. Suppose $e^{i \theta_{0}}$ is not a limit point of the zeros $\left\{a_{k}\right\}$. Then, there exists a positive number $p$ such that there are no zeros $\left\{a_{k}\right\}$ in $\Delta^{\prime}=\left\{z|| z|<1| z-,e^{i \theta_{0}} \mid<p\right\}$. Thus, by theorem $\mathrm{A}, B^{*}\left(e^{i \theta}\right)$ exists and is of modulus 1 for each $e^{i \theta}$ on the boundary of $\Delta^{\prime}$. But, by a theorem of Lohwater [5, p. 153], since $P=e^{i \theta_{0}}$ is a singular point for $B(z)$, there exists a point $e^{i \theta^{*}}$ on the boundary of $\Delta^{\prime}$ such that $B^{*}\left(e^{i \theta^{*}}\right)=0$. This is a contradiction. Therefore, $e^{i \theta_{0}}$ is a limit point of $\left\{a_{k}\right\}$. This completes the proof of theorem 4 .

Remark. We point out that theorem 3 and theorem 4 can be modified in the obvious way to give necessary and sufficient conditions for $B(z)$ to have left-sided limit of modulus 1 at $e^{i \theta_{0}}$.

Acknowledgment. The author is grateful to Professor W. Seidel for several valuable conversations concerning theorem 3 .

\section{REFERENCES}

[1] A. P. Calderón, A. González-Domínguez, and A. Zygmund, Nota sobre los valores limites de funciones analiticas, Revista de la Unión Matemática Argentina, 14 (1949), pp. 16-19.

[2] E. F. Collingwood and A. J. Lohwater, The Theory of Cluster Sets, Cambridge University Press, New York, 1966.

[ 3 ] O. Frostman, Sur les produits de Blaschke, Kungl. Fysiogr. Sällsk. i Lund Förh., Bd. 12, Nr. 15 (1942), pp. 169-182. 
[4] A. J. Lohwater, The boundary values of a class of meromorphic functions, Duke Math. J. 19 (1952), pp. 243-252.

[ 5 ] A. J. Lohwater, On the Schwarz reflection principle, Mich. Math. J. 2 (1953-54), pp. 151-156.

[6] R. Nevanlinna, Analytic Functions, Springer-Verlag, New York, 1970.

[ 7 ] S. Saks, Theory of the Integral, 2nd Ed., Dover Publications, Inc., New York, 1964.

[ 8 ] W. Seidel, On the distribution of values of bounded analytic functions, Trans. Amer. Math. Soc. 36 (1934), pp. 201-226.

[9] A. Zygmund, Trigonometric Series, Vol. 1, 2nd Ed., Cambridge University Press, New York, 1959.

Oklahoma State University 